\title{
Correlated response to selection during selfing
}

\author{
E. M. TOMS, M. J. KEARSEY* \& M. A. CORNISH \\ School of Biological Sciences, University of Birmingham, Birmingham B15 2TT, UK
}

\begin{abstract}
Theory for predicting the correlated response to selection during selfing is proposed and then validated by computer simulation. Pairs of characters, correlated either by pleiotropy or linkage, were simulated; linkage was in coupling, repulsion or a combination of the two. The correlated responses to both family and sib selection were examined. Comparison of expected with the average observed correlated responses showed the accuracy of the theory, but the observed sampling variation indicated that a wide variability of individual responses is to be expected in practice.
\end{abstract}

Keywords: correlated response, family selection, linkage, selfing, sib selection, pleiotropy.

\section{Introduction}

The improvement of one character by selection frequently causes simultaneous changes in other characters. The effect is the result of correlations between characters, which may be genetic or environmental in nature. Genetic correlations arise from pleiotropy, from linkages between loci controlling the characters or from random genetic drift. According to Falconer (1989) and Simmonds (1979), pleiotropy is the chief cause of genetic correlations, while Mather \& Jinks (1982) have argued that linkage is the more likely explanation. A subsequent study by Jinks et al. (1985) indicated that either or both factors may be important, depending on the pair of characters considered.

If the correlation is heritable, i.e. additive genetic, then the correlated response may be predicted from an earlier generation. The response is measured by the change in the mean of the distribution of lines for the correlated character compared with the distribution of lines derived without any selection. The theory describing the correlated response to selection in a random mating population is described by Falconer (1989). Unfortunately, equivalent deterministic theory has not yet been developed for predicting the correlated response within an inbreeding population. It may be argued, however, that the formula for predicting the correlated response within a random mating population may be applied to an inbreeding population, in the same way that the formulae for predicting the direct

*Correspondence. response within a selfing programme take the same form as those derived for a random mating population (Cornish, 1990a; Toms et al., 1994). Thus, it is proposed that the effect of a single round of family selection, imposed on character $\mathrm{X}$ at the $\mathrm{F}_{N}$ generation, on the mean of a correlated character $\mathrm{Y}$ may be predicted by:

$C R_{Y}=i h_{X} r_{A} \sigma_{A_{Y}}$,

where $i$ is the intensity of selection on character $\mathrm{X}, h_{X}$ is the square root of the heritability of character $\mathrm{X}, r_{A}$ is the additive genetic correlation between characters $\mathrm{X}$ and $\mathrm{Y}$, and $\sigma_{A_{Y}}$ is the additive genetic standard deviation of character $\mathrm{Y}$, all based on $\mathrm{F}_{N}$ family means. As for the direct response, the correlated response is measured on the population of inbred lines derived from the families selected at the $F_{N}$ generation (Toms et al., 1994). Thus the mean of this distribution is predicted by $m_{Y} \pm C R_{Y}$, where $m_{Y}$ is the midparental value of character $Y$, in the notation of Mather \& Jinks (1982). With the exception of the additive genetic correlation, the components in these formulae are estimated from analyses of the means and variances carried out independently for characters $\mathrm{X}$ and $\mathrm{Y}$. A general formula for estimating the narrow heritability is given by Toms et al. (1994).

The additive genetic correlation is estimated by partitioning the observed phenotypic correlation into genetic and environmental components, which may be achieved by an analysis of covariance (Steel \& Torrie, 1981; Falconer, 1989). The between-family correlation which can be extracted from the analysis represents the 
additive genetic correlation $\left(r_{A}\right)$ in the absence of dominance and between-family environmental effects:

$r_{A}=\frac{\sigma_{A_{X Y}}}{\sqrt{\sigma_{A_{X}}^{2} \sigma_{A_{Y}}^{2}}}$,

where $\sigma_{A_{X Y}}$ the additive genetic covariance between characters $\mathrm{X}$ and $\mathrm{Y}$, and $\sigma_{A_{X}}^{2}$ and $\sigma_{A_{Y}}^{2}$ are the additive genetic variances of characters $\mathrm{X}$ and $\mathrm{Y}$, respectively.

The validity of the above formulae for predicting the correlated response to selection during selfing was assessed by computer simulation.

\section{Method}

A selfing and selection programme was simulated using a computer program for modelling plant breeding situations (Cornish, 1990a,b). Two inbred parents were crossed to generate an $F_{1}$ hybrid. This $F_{1}$ was selfed to produce $1000 \quad F_{2}$ individuals from which, after repeated selfing, $1000 \mathrm{~F}_{6}$ lines were derived by the method of single seed descent. At either the $\mathrm{F}_{3}$ or $\mathrm{F}_{4}$ generation, selection for low score was applied to one character (character $\mathrm{X}$ ), based on families of size one. The correlated response was assessed at the $F_{6}$ generation on the correlated character (character $\mathrm{Y}$ ), again based on families of size one. Both family and sib selection, as described by Toms et al. (1994), were simulated. Independent runs of the simulation were performed for a range of selection intensities from 1 per cent to 100 per cent, and each simulation was repeated 200 times.

Correlations between characters due to pleiotropy and linkage were simulated separately.

\section{Correlation resulting from pleiotropy}

Two identically controlled characters correlated as a result of pleiotropy were simulated: character $X$ was controlled by 20 unlinked loci, character $\mathrm{Y}$ was similarly controlled by 20 unlinked loci, 10 of these loci being common to both characters. All of the loci contributed equal additive effects, dominance and epistasis were not included, and the environmental variance was set so that the narrow heritability of each character was 50 per cent.

Calculation of the expected correlated response required knowledge of the additive genetic correlation of family means between the two simulated characters. The additive genetic correlations appropriate for calculating the correlated responses to family and sib selection are defined in terms of the individual additive genetic effects in Table 1. Substitution of the appropriate values revealed that the additive genetic correla- tion between these two characters was 0.5 , for both $F_{3}$ and $\mathrm{F}_{4}$ family means, and for both family and sib selection.

\section{Correlation resulting from linkage}

Two characters with identical control, correlated as a result of linkage between the loci affecting the characters, were also simulated. A total of 30 loci were involved, linked in pairs, with a recombination frequency of 0.25 between each pair of genes. This intermediate value was chosen for the recombination frequency because the effect of linkage on the variances is greatest at such values (Mather \& Jinks, 1982). For each linked pair, one gene affected character $\mathrm{X}$, while the other affected character $\mathrm{Y}$, so that character $X$ was controlled by 15 unlinked loci and character $\mathrm{Y}$ was similarly controlled by 15 unlinked loci. Three different linkage combinations were simulated: (i) all pairs linked in coupling; (ii) all pairs linked in repulsion; (iii) eight pairs linked in coupling and seven pairs in repulsion. Each of the loci contributed equal additive effects, and dominance and epistasis were not included. The environmental variance was set so that the narrow heritability of each character was 50 per cent.

Linkage has no effect on generation means in the absence of epistasis, but its effect is evident in the variances and covariances (Mather \& Jinks, 1982). To determine the additive genetic correlation between the two simulated characters the effect of linkage on the additive genetic variances was first considered. This effect has been discussed in detail by Jinks \& Pooni (1976, 1982), Kearsey (1985) and Mather \& Jinks (1982). In brief, in the presence of linkage the expectation of the additive genetic variance $(D)$ is dependent on the rank $(r)$ of the variance:

$D_{r}=\sum_{i=1}^{k} d_{i}^{2} \pm c_{k}^{k-1} \sum_{i=1}^{k} \sum_{j=i+1}^{k} 2\left(1-2 p_{i j}\right)^{r} d_{i} d_{j}$,

using the notation of Mather \& Jinks (1982). The linkage component $2\left(1-2 p_{i j}\right)^{\prime} d_{i} d_{j}$ is added for each pair of genes linked in coupling $(C)$ and subtracted for each pair linked in repulsion $(R)$, where $p_{i j}$ is the recombination frequency between the $i$ th and $j$ th loci. It is only this linkage component that contributes to the additive genetic covariance between the two characters, and it does so in the proportions expected for the generation and rank of the covariance being considered. The additive genetic variances of each character are not affected by linkage because the loci controlling each character alone are unlinked. These variances and covariances were used to define the additive genetic correlation in terms of the individual 
additive genetic effects in Table 2. Calculation of the additive genetic correlations for the three linkage combinations considered indicated that, in the case of linkage, the additive genetic correlation between the two characters appropriate for predicting the correlated response is dependent on the linkage relationship, the generation of selection and also the type of selection imposed.

Predicted responses were calculated using the formulae presented above. Values of $i$ were obtained from the tables given by Falconer (1989) for selection from populations larger than 100 . The observed correlated response was measured by the difference in mean of character $\mathrm{Y}$ of the selected and unselected lines, averaged over the 200 repeat runs. The variability of individual runs was examined by determining the intervals including 95 per cent of the observations.

\section{Results and Discussion}

\section{Correlation resulting from pleiotropy}

The expected and observed responses measured by the mean of the correlated character $\mathrm{Y}$ following selection for character $\mathrm{X}$ at the $\mathrm{F}_{3}$ or $\mathrm{F}_{4}$ generation are compared in Fig. 1. The plots show a very close agree-

Table 1 Definition of the additive genetic correlation $\left(r_{A}\right)$ of $F_{3}$ and $F_{4}$ family means between two characters correlated as a result of pleiotropy, appropriate for predicting the correlated response to family or sib selection. Definition is in terms of the individual additive genetic effects $\left(d_{x}\right.$ and $\left.d_{y}\right)$ of the pleiotropic genes on characters $\mathrm{X}$ and $\mathrm{Y}$, respectively, and the family size $(n)$

\section{Family}

$$
\frac{1}{2} \Sigma d_{x} d_{y}+\frac{1}{n}\left(\frac{1}{4} \Sigma d_{x} d_{y}\right)
$$

$F_{3}$ family means

$F_{4}$ family means
Sib

$$
\begin{gathered}
\frac{\frac{1}{2} \Sigma d_{x} d_{y}}{\sqrt{\left[\frac{1}{2} \Sigma d_{x}^{2}\right]\left[\frac{1}{2} \Sigma d_{y}^{2}\right]}} \\
\frac{\frac{3}{4} \Sigma d_{x} d_{y}}{\sqrt{\left[\frac{3}{4} \Sigma d_{x}^{2}\right]\left[\frac{3}{4} \Sigma d_{y}^{2}\right]}}
\end{gathered}
$$

Table 2 Definition of the additive genetic correlation $\left(r_{A}\right)$ of $F_{3}$ and $F_{4}$ family means between two characters correlated as a result of linkage, appropriate for predicting the correlated response to family or sib selection. Definition is in terms of the individual additive genetic effects $\left(d_{i}\right.$ and $\left.d_{j}\right)$ of the linked genes on characters $\mathrm{X}$ and $\mathrm{Y}$, respectively, the recombination frequency $(p)$, and the family size $(n) . D_{X}$ and $D_{Y}$ are the additive genetic variances of characters $X$ and $Y$, respectively, where $D_{X}=\Sigma d_{i}^{2}$ and $D_{Y}=\Sigma d_{j}^{2}$

$F_{3}$ family means

$F_{4}$ family means

$$
\begin{gathered}
\frac{\Sigma\left[\frac{1}{2}(1-2 p)+\frac{1}{n} \frac{1}{4}(1-2 p)^{2}\right] d_{i} d_{j}}{\sqrt{\left[\frac{1}{2} D_{X}+\frac{1}{n}\left(\frac{1}{4} D_{X}\right)\right]\left[\frac{1}{2} D_{Y}+\frac{1}{n}\left(\frac{1}{4} D_{Y}\right)\right]}} \\
\frac{\Sigma\left[\frac{1}{2}(1-2 p)+\frac{1}{4}(1-2 p)^{2}+\frac{1}{n} \frac{1}{8}(1-2 p)^{3}\right] d_{i} d_{j}}{\sqrt{\left[\frac{3}{4} D_{X}+\frac{1}{n}\left(\frac{1}{8} D_{X}\right)\right]\left[\frac{3}{4} D_{Y}+\frac{1}{n}\left(\frac{1}{8} D_{Y}\right)\right]}}
\end{gathered}
$$

$$
\begin{gathered}
\frac{\Sigma \frac{1}{2}(1-2 p) d_{i} d_{j}}{\sqrt{\left[\frac{1}{2} D_{X}\right]\left[\frac{1}{2} D_{Y}\right]}} \\
\frac{\Sigma\left[\frac{1}{2}(1-2 p)+\frac{1}{4}(1-2 p)^{2}\right] d_{i} d_{j}}{\sqrt{\left[\frac{3}{4} D_{X}\right]\left[\frac{3}{4} D_{Y}\right]}}
\end{gathered}
$$


Fig. 1 Comparison of the expected and observed means of character $\mathrm{Y}$, of the $F_{6}$ lines derived from the $F_{3}$ or $F_{4}$ generation following family or $\mathrm{sib}$ selection for character $X$, where the simulated characters $\mathrm{X}$ and $\mathrm{Y}$ are correlated due to pleiotropy. The expected values are given by the continuous lines, the observed values are shown by the points, and for comparison the direct responses are indicated by the broken lines. The intervals including 95 per cent of the observations are also shown.

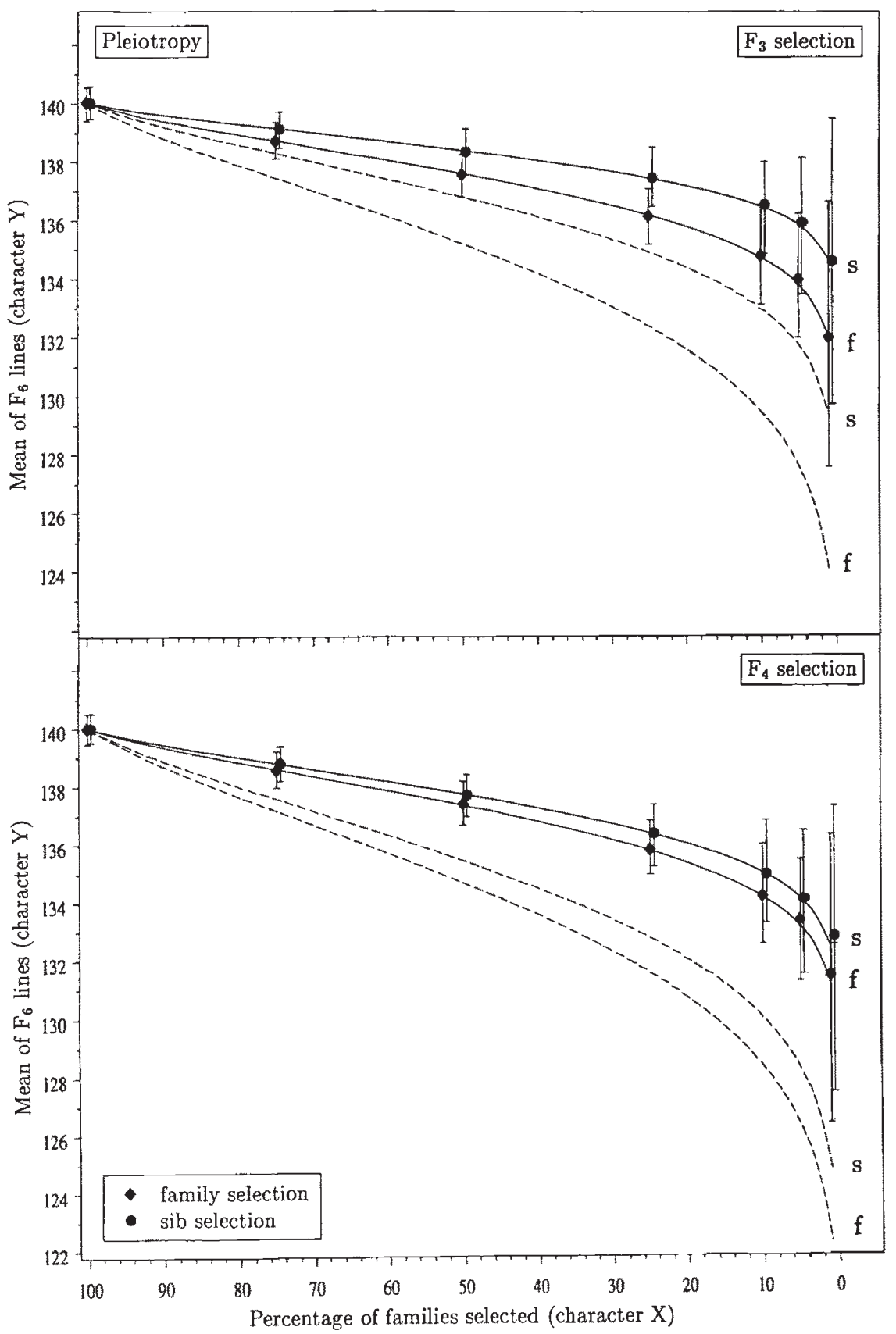

ment between the expected and observed correlated responses, for both family and sib selection. The observed 95 per cent confidence intervals, also indicated in these graphs, revealed a large variability of individual runs, and the limits of this variability expanded quite dramatically with increasingly intense selection. Comparing the correlated and direct response (also shown in Fig. 1) indicates that the magnitude of the correlated response was half that of the direct response. This corresponded to an additive genetic correlation of 0.5 between the two characters.

\section{Correlation resulting from linkage}

The expected and observed correlated responses resulting from coupling linkages are plotted in Fig. 2, the responses due to repulsion linkages are shown in Fig. 3 and, finally, the correlated responses resulting from a combination of coupling and repulsion linkages are presented in Fig. 4. The observed 95 per cent confidence intervals are also indicated in these graphs. The very close agreement of the observed means of the correlated character with those expected, for all of the 


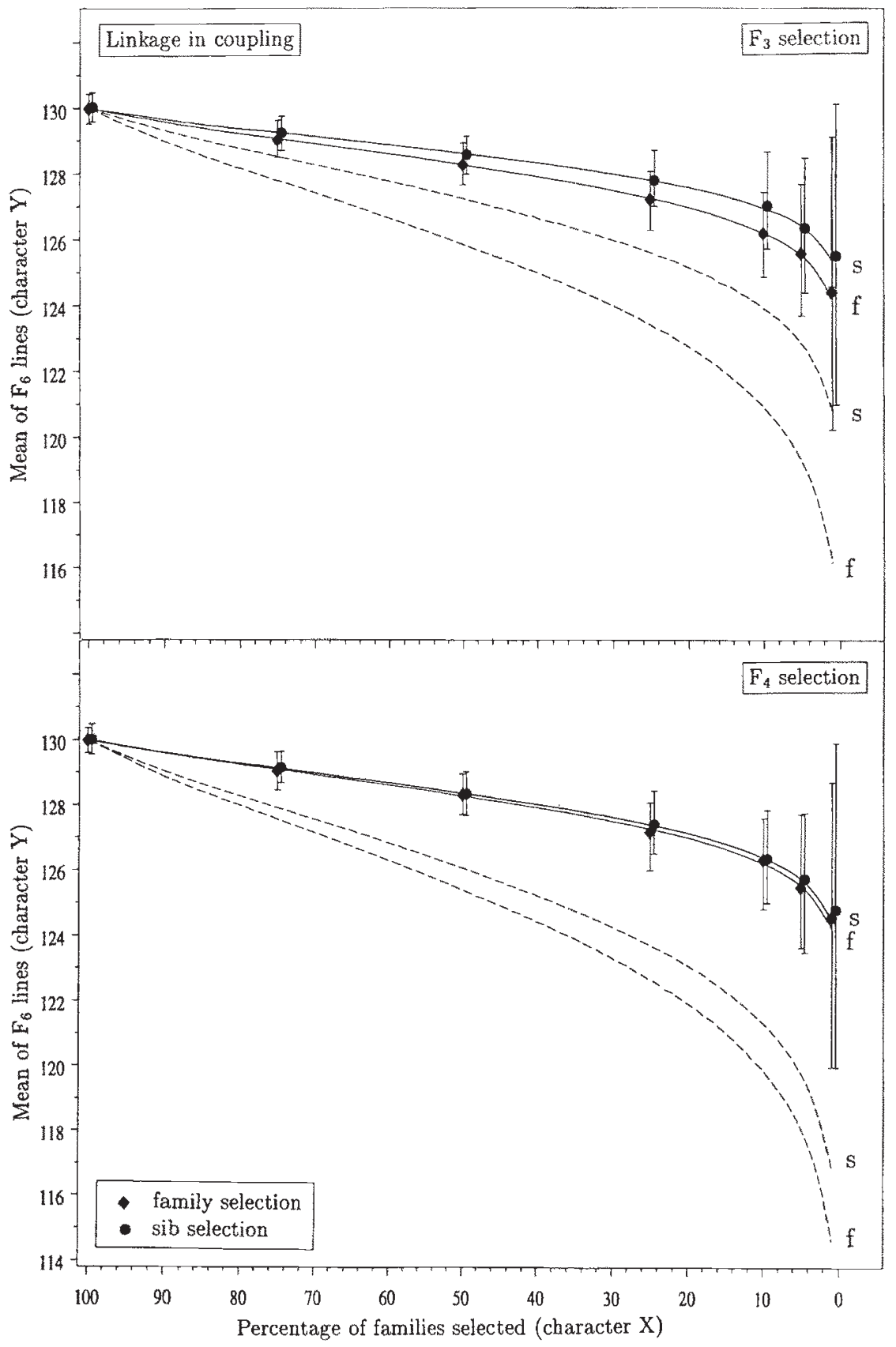

Fig. 2 Comparison of the expected and observed means of character $\mathrm{Y}$, of the $F_{6}$ lines derived from the $F_{3}$ or $F_{4}$ generation following family or sib selection for character $X$, where the simulated characters $\mathrm{X}$ and $\mathrm{Y}$ are correlated due to linkage in coupling. The expected values are given by the continuous lines, the observed values are shown by the points, and for comparison the direct responses are indicated by the broken lines. The intervals including 95 per cent of the observations are also shown. linkage combinations considered, was convincing evidence of the accuracy of the predictive theory. Nevertheless, the confidence intervals indicated considerable variability of individual observations about these means, particularly under intense selection. Comparison of the results for the three linkage combinations shows that the direction of the correlated response is dictated by the linkage phase of the loci controlling the characters. When the pairs of loci were all linked in coupling, the correlated response was in the same direction as the direct response. Thus, in Fig. 2 the correlated response of character $Y$ was for decreased score as selection on character $\mathrm{X}$ was for low score. The opposite trend is apparent in Fig. 3, where the correlation between characters resulted from repulsion linkages between their loci. In this case, 
Fig. 3 Comparison of the expected and observed means of character $\mathrm{Y}$, of the $F_{6}$ lines derived from the $F_{3}$ or $F_{4}$ generation following family or sib selection for character $X$, where the simulated characters $\mathrm{X}$ and $\mathrm{Y}$ are correlated due to linkage in repulsion. The expected values are given by the continuous lines and the observed values are shown by the points. The intervals including 95 per cent of the observations are also indicated.

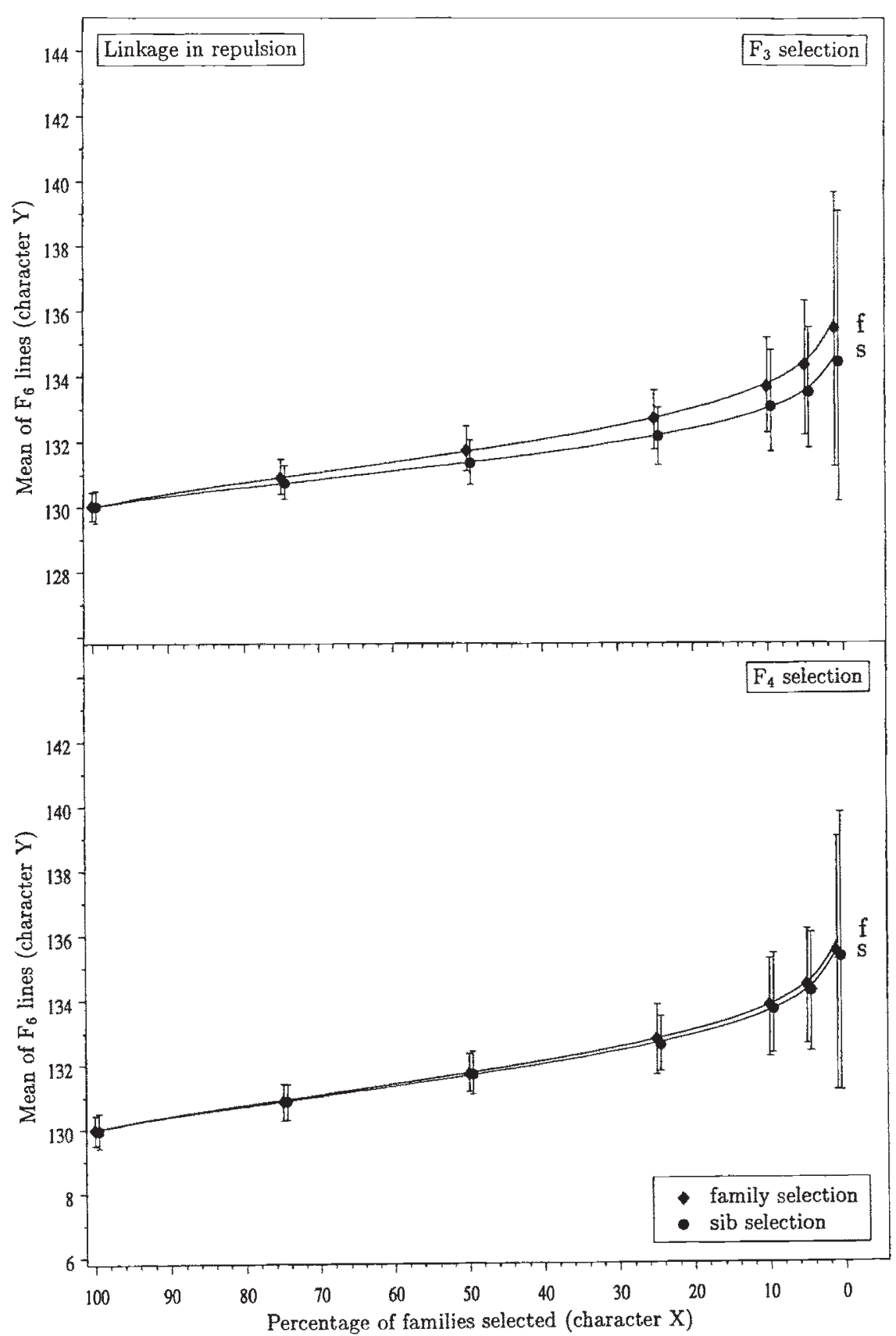

selection for low score on character $\mathrm{X}$ resulted in a correlated increase in the mean for character $Y$. Finally, Fig. 4 indicates that the effects of coupling and repulsion linkages counteracted each other. A very slight correlated response was evident as eight pairs were linked in coupling and only seven in repulsion.

The magnitude of the correlated response relative to the direct response (also shown in Fig. 2) varied depending on the linkage relationship, and the generation and type of selection. This corresponded to the magnitude of the additive genetic correlation between the two characters.

Trends common with those shown by the direct selection response were also evident. The correlated response to $F_{4}$ selection was greater than to $F_{3}$ selection. Family selection effected a larger correlated 


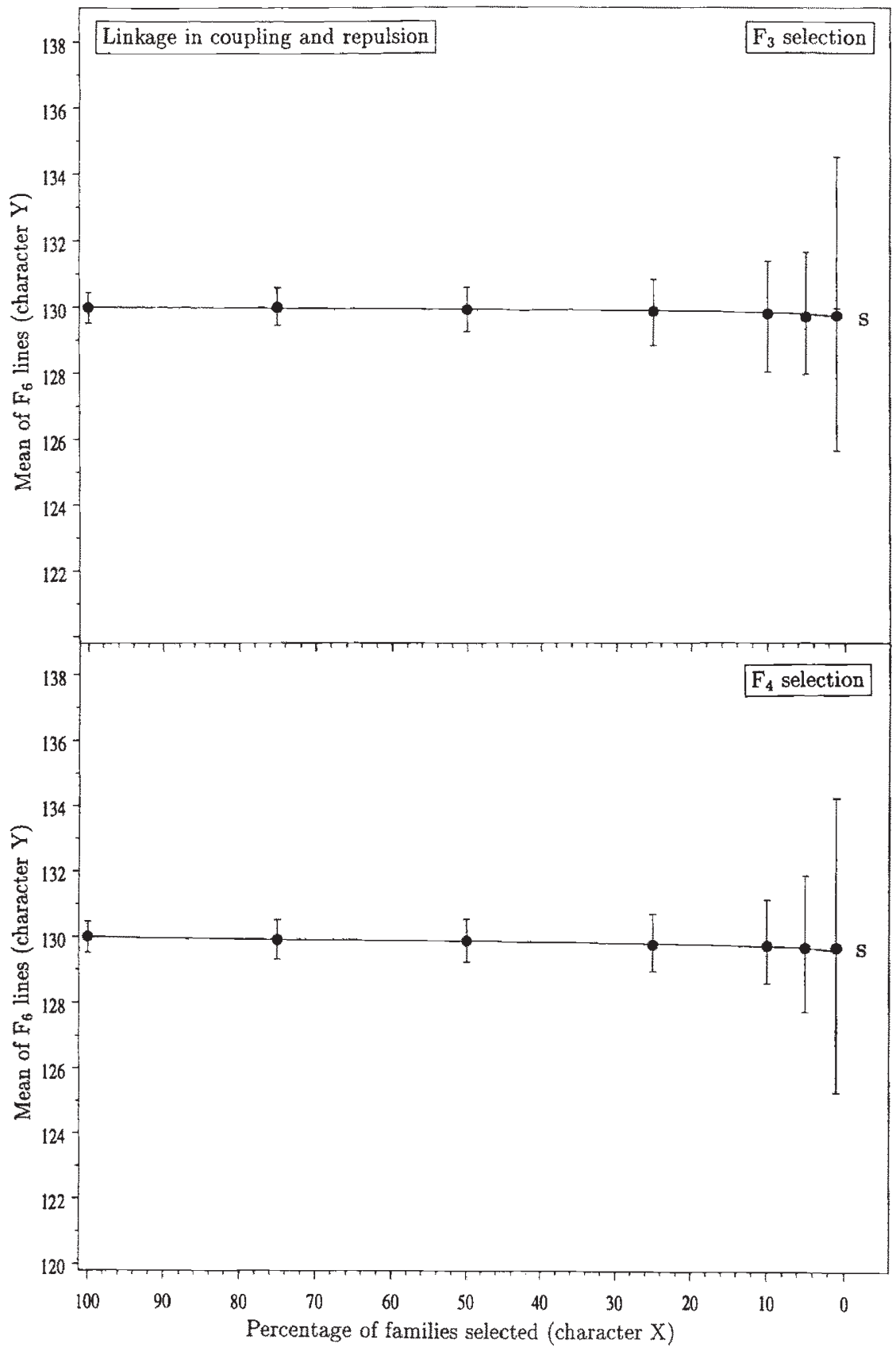

Fig. 4 Comparison of the expected and observed means of character $Y$, of the $\mathrm{F}_{6}$ lines derived from the $\mathrm{F}_{3}$ or $\mathrm{F}_{4}$ generation following sib selection for character $\mathrm{X}$, where the simulated characters $\mathrm{X}$ and $\mathrm{Y}$ are correlated due to linkage in coupling and repulsion. The expected values are given by the continuous lines and the observed values are shown by the points. The intervals including 95 per cent of the observations are also indicated. The response to family selection is almost identical, and is not shown. response than sib selection but the difference between the two was marginal at the later generation. Taking into account the substantial overlap of the confidence intervals observed for family and sib selection, it is clear that individual observations would almost certainly be indistinguishable in practice.

\section{Conclusions}

The response equations for predicting the correlated response to family selection and sib selection during selfing were validated for a limited set of conditions. Predictions of the correlated responses resulting from pleiotropy and from linkage were equally accurate. The results supported the proposal that equivalent theory to that describing the correlated response in a random mating population (Falconer, 1989) may be applied to an inbreeding population.

Nevertheless, the wide variability of individual responses undermines the value of very precise prediction of the correlated response to selection. In practice, predictions of correlated response are liable to larger 
sampling errors than the direct response because estimates of the additive genetic correlation are seldom precise (Falconer, 1989). Consequently, the predictive theory may be most useful in applications where less precision is required. For example, in cross prediction, use of the theory may aid the choice between crosses based on the effects of selection on correlated characters. Alternatively, the theory may be used to aid the design of optimal breeding programmes, as Cornish (1990b) suggests for single characters.

Ideally, further simulation studies would be useful to substantiate the theory for a wider range of characters and conditions, since various effects were not considered that would likely be present in practice. These include the effects of dominance, non-allelic interactions, linkage of loci controlling a single character, genes of unequal effects and, lastly, the presence of genotype $\times$ environmental interaction.

\section{References}

CORNISH, M. A. 1990a. Selection during a selfing programme. I. The effects of a single round of selection. Heredity, $\mathbf{6 5}$, 201-211.
CORNISH, M. A. $1990 \mathrm{~b}$. Selection during a selfing programme. II. The effects of two or more rounds of selection. Heredity, 65, 213-220.

FALCONER, D. S. 1989. Introduction to Quantitative Genetics, 3rd edn. Longman Scientific \& Technical, Harlow.

JINKS, J. L AND POONI, H. S. 1976. Predicting the properties of recombinant inbred lines derived by single seed descent. Heredity, 36, 253-266.

JINKS, J. L. AND POONI, H. S. 1982. Predicting the properties of pure breeding lines extractable from a cross in the presence of linkage. Heredity, 49, 265-270.

JINKS, J. L., POONI, H. S. AND CHOWDHURY, M. K. U. 1985. Detection of linkage and pleiotropy between characters of Nicotiana tabacum using inbred lines produced by dihaploidy and single seed descent. Heredity, 55, 327-333.

KEARSEY, M. J. 1985. The effect of linkage on additive genetic variance with inbreeding an $\mathrm{F}_{2}$. Heredity, 55, 139-143.

MATHER, K. AND JINKS, J. L. 1982. Biometrical Genetics, 3rd edn. Chapman \& Hall, London.

simmonds, N. W. 1979. Principles of Crop Improvement. Longman Group, London.

STEEL, R. G. D. AND TORRIE, J. H. 1981. Principles and Procedures of Statistics, 2nd edn. McGraw-Hill, Singapore.

TOMS, E. M., CORNISH, M. A. AND KEARSEY, M. J. 1994. Comparison of family and sib selection during selfing. Heredity, 73, 635-641. 